\title{
SPLIT: A sound editor for a PDP-8 computer
}

\author{
STEVEN M. MYEROW \\ Laboratory for Nuclear Science, Massachusetts Institute of Technology \\ Cambridge, Massachusetts 02139 \\ and \\ RICHARD B. MILLWARD \\ Brown University, Providence, Rhode Island 02912
}

\begin{abstract}
SPLIT is a program that helps prepare sounds for use in psychological experiments. Analog signals are converted to digital form and stored on a disk in real time. Digital sound files can be viewed on an oscilloscope display and played back for on-line auditory inspection. Interactive commands allow the user to segment and combine sounds with precise temporal control.
\end{abstract}

SPLIT is a waveform editor for the PDP-8/E computer which manipulates digitized signals much as a text editor processes characters. Analog signals, usually sounds, can be sampled in real time with 8-bit accuracy at rates up to $21 \mathrm{kHz}$ and stored as digital files on a disk. The aggregate of digital files on the disk may contain up to 1.6 million samples (160 sec of sound at $10 \mathrm{kHz}$ ), but a single file is limited to 800,000 samples. Files may be split into segments or combined to form new files by special SPLIT commands. Disk files are converted to analog signals to be played back through speakers or headphones and are presented graphically on an oscilloscope display. An alphanumeric TV display supplies file status information throughout the course of an editing session. SPLIT's power resides in the variety of control functions for sound segmentation and in the three forms of immediate feedback while editing: auditory playback, graphic waveform display, and quantitative information on a TV monitor.

SPLIT is mostly used as a preprocessor to prepare stimuli for later use. For example, a user might record a sequence of sounds from audio tape into a large digitized sound file on the disk. Then, using SPLIT's interactive editing facilities, portions of interest can be quickly isolated and separate stimulus files made for each segment. The experimenter has precise control over the start, end, and length of each stimulus - right down to the individual $A / D$ sample. After segmenting the sounds, multiple recordings of stimulus items can be easily compared in order to select the best ones. At this point, SPLIT's task is complete.

SPLIT does not synthesize sounds, is not designed to make precise stimulus tapes, and cannot run an experiment; the experimenter normally uses other programs to perform these functions. Nor does it analyze sounds, although SPLIT has been used as a tool for speech analysis (e.g., visual determination of voice onset times and fundamental frequency).
SPLIT is built from functionally independent modules that may be used separately in assembly language (PAL8) or BASIC programs. With these modules, it is easy to write general or special-purpose programs to generate complex sound sequences from the prepared stimulus files. On-line experiments to present the stimuli and record responses and response times may also be quickly programmed. SPLIT has other uses too: For example, the system can be used to play computer synthesized music and as an aid in music transcription.

A variety of computer-controlled systems to process sound stimuli have been implemented. The S.I.M.P.L.E. system (Aaronson, Grupsmith, \& Ohman, 1975) produces sound stimulus tapes from an audio library tape without digitizing the sounds. The addition of $A / D$ and $\mathrm{D} / \mathrm{A}$, as in SPLIT, increases the experimenter's control over individual sound segments and also allows the computer to synthesize stimuli. Some systems use core memory for real-time sound storage, with digital magnetic tape as a non-real-time storage medium (Knight and Kantowitz, 1973; Poltrock \& Mathews, 1976). The maximum length of a sound segment is limited by core memory size in these systems. SPLIT's use of a disk as a real-time sound storage device allows convenient processing of large sound segments. A larger computer system using both disk and magnetic tape for real-time storage is described in Nakatani (1977). It has some features not available in SPLIT, but is similar in concept.

\section{HARDWARE}

The SPLIT hardware is diagrammed in Figure 1. SPLIT and its supporting programs use $28 \mathrm{k}$ of memory on the PDP-8/E computer. However, the system can be converted to run in $16 \mathrm{k}$ without extensive modifications. The RK8-E moving-head disk holds 1.6 million 12-bit words on a removable cartridge. The DEC AX08 laboratory peripheral includes $A / D$ and $D / A$ converters 


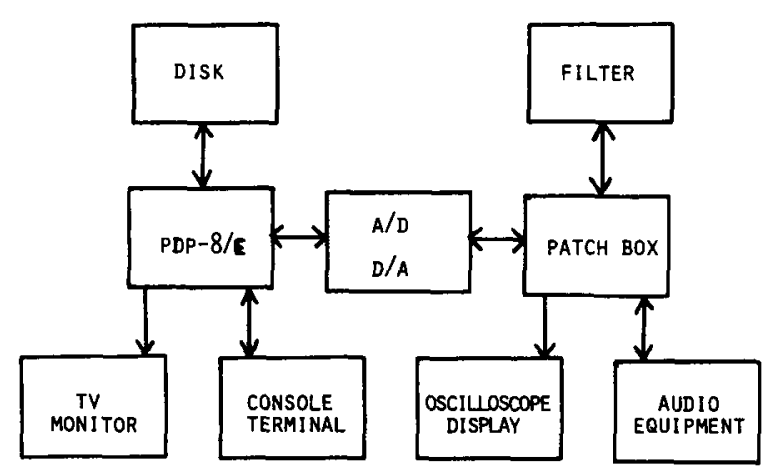

Figure 1. SPLIT hardware.

and two real-time clocks. The two D/A channels are both used for audio output and graphical oscilloscope display, making simultaneous audio and video output impossible. A reed relay in the patch box selects the output destination (audio or video) and is computer controlled through the AX08. A Krohn-Hite adjustable bandpass filter decreases foldover and other noise on recording and playback. The audio equipment includes a tape deck, stereo receiver, speakers, and headphones. The equipment can be made to support two-channel signal processing, but the software currently handles only monaural sound.

\section{OPERATING SPLIT}

A SPLIT user controls the program by a combination of acts: typing commands, turning knobs, and setting toggle switches. There are three modes of operation: recording, audio, and visual. The recording mode is used to enter analog signals into the computer. The operator first sets the rate of sampling and selects a file name. Next the source volume is adjusted in order to obtain good dynamic range while minimizing clipping. The user throws a toggle switch to enter recording mode. SPLIT samples the signal without storing it and simultaneously displays on the TV monitor accumulated results of the maximum unclipped amplitude and number of clipped samples. (Samples with full-scale amplitude are categorized as clipped.) The amplitude reset (A) command clears these results to zero and restarts the calculations. The experimenter samples the input source, modifying the volume and using the $A$ command when necessary, until a satisfactory signal level is attained. Recording onto the disk is begun by the INPUT (I) command. INPUT terminates when another key is typed, or if the disk fills up. The user may then enter either audio mode, to hear the recorded signal, or visual mode, to see the waveform. If unacceptable, the sound may be recorded again, erasing the previous file.

In audio mode, SPLIT plays sound segments through speakers or headphones in one of three ways: contin- uous repetition (repeat mode), short delays between repetitions (play mode), and single play initiated by a terminal keystroke (once mode). In visual mode, the samples in sound segments are displayed on the oscilloscope. The user may adjust the number of points plotted from 2 to 500 . Since the points are evenly spaced across the screen, decreasing the number of points on the display enlarges the picture of a waveform-a useful function because even a pure sine wave might look like random noise if the point density on the screen was too great. If a sound segment contains more samples than allowed on the screen, the waveform picture will be divided by parallel lines into halves, displaying the beginning and end portions of the segment (see Figure 2). Switching between audio and visual modes is accomplished by simply pressing the ESC key on the console. The once mode $(0)$ command is also a bridge between audio and visual modes since it enters audio mode, plays the signal once, and then returns to visual mode.

\section{Sound Segmentation}

A playback window is a viewport into a waveform segment. A window, which can include the entire file or any portion of it, is analogous to the viewing screen on a home-movie editor (see Figure 3). Editing is performed
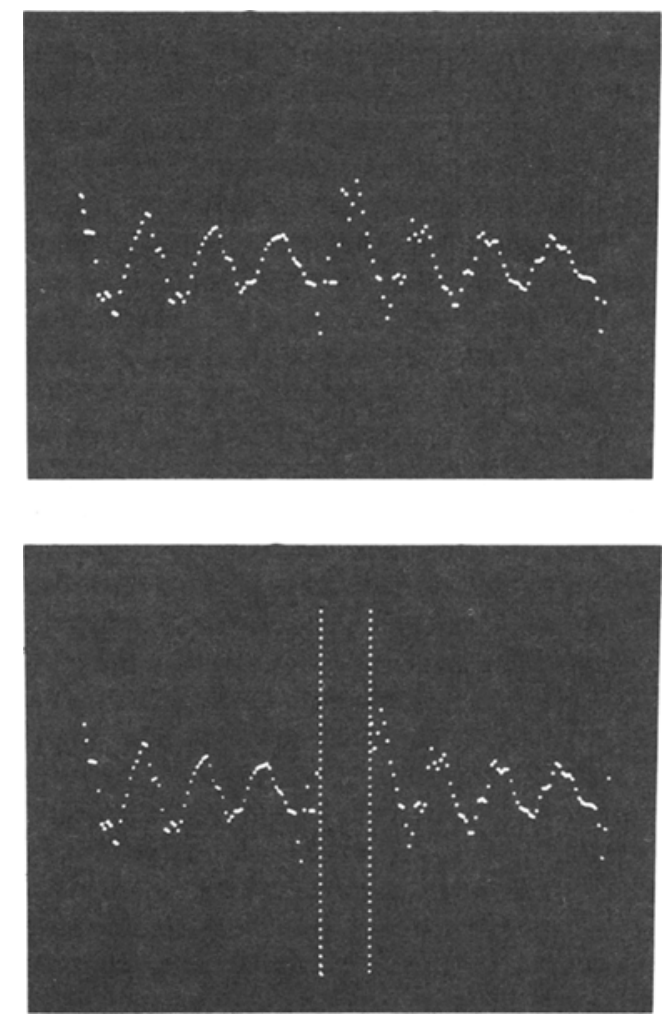

Figure 2. Oscilloscope displays: In the bottom picture, the window contains more samples than the user adjustable screen limit. 


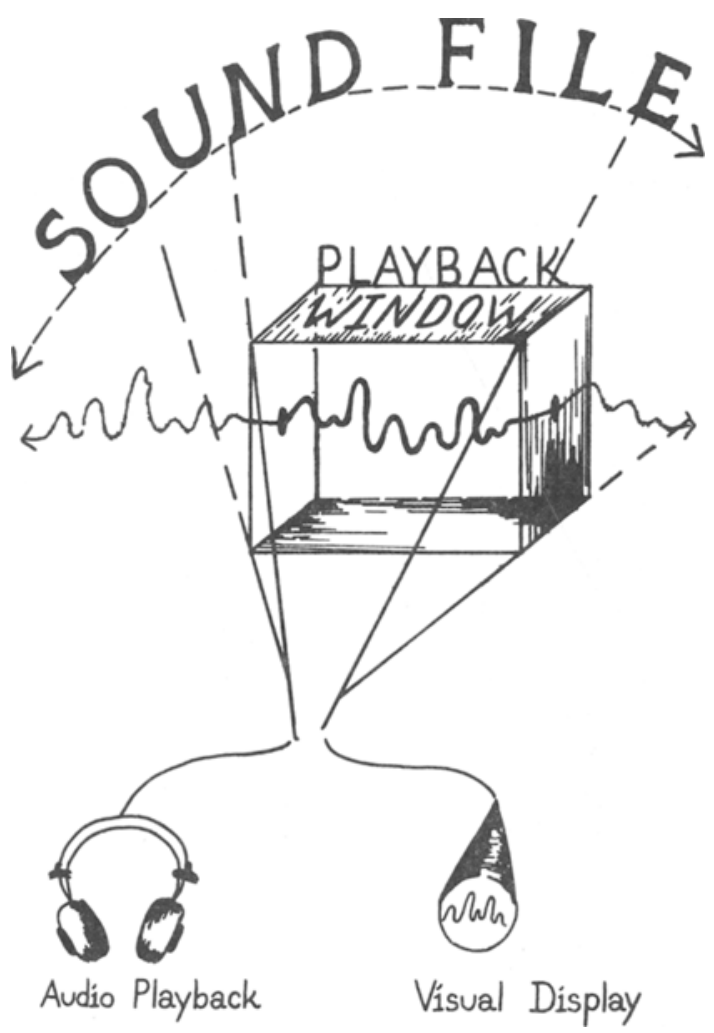

Figure 3. Pictorial description of a playback window.

by adjusting the position and size of windows. The sound segment in a window can be deleted, stored in a separate file, or concatenated with other segments. Playback windows are defined by the position of their endpoints. The endpoints may be moved in either audio or visual modes by turning knobs or by specifying their positions quantitatively with typed commands. Also, in audio mode, the endpoint locations may be marked in real time as the sound is played by striking terminal keys at the desired moments. As the window changes, the TV monitor display is instantly updated to reflect the current window parameters: the position of the front pointer, the position of the back pointer, and the window length (see Figure 4).

The four variable reference knobs used for editing are part of the AX08 lab peripheral. Knob 0 moves the entire window through the file without changing the window's length. Knob 1 repositions the front of the window, keeping the back stationary and changing window length. Knob 2 moves the back of the window, keeping the front stationary. Knob 3 sets a scale factor that determines the amount of window movement caused by incremental rotations of the other three knobs. Using these knobs, continuous adjustments to the window can be heard and seen at slow or fast rates. While in visual mode, knob rotations cause almost instantaneous window movement, apparent on both the oscilloscope and TV displays. In play mode and once
OUTPUT: BU SAMPLES 0

ACTIVE PLAYBACK: 1) BI SAMPLES 2300

$\begin{array}{lll}\text { FRONT } & 288 & 28.80 \mathrm{msec} \\ \text { BACK } & 438 & 43.80 \mathrm{msec} \\ \text { LENGTH } & 151 & 15.10 \mathrm{msec}\end{array}$

SCALE $\quad 32$

RATE $\quad 10000$

GRAPH: $\quad 150$

PLAYBACK FILES:
1) $\mathrm{BI}$
2) BAE
3)BE

Figure 4. TV monitor display: The active playback window is from the file BI, whose total length is 2,300 samples. FRONT, BACK, and LENGTH describe the position and size of the window; that is, the window begins at sample 288, which is $28.80 \mathrm{msec}$ from the start of the file (calculated from the currently selected sampling rate of $10 \mathrm{kHz}$ ). Any of the playback files may be inspected by typing their identification number.

mode, window changes are only made between playback repetitions. However, $\mathrm{P}$ or $\mathrm{O}$ may be typed at any time to immediately stop playback, read the control knobs and change the window accordingly, and restart playback in the respective mode. In repeat mode, SPLIT is totally dedicated to sound playback and $\mathrm{R}$ must be typed to briefly interrupt the output and allow knob changes to affect the window. The knobs may be turned only a fixed amount in either direction. If the end of travel is reached, a switch may be set to "disconnect" the knobs from the program. Now the knobs may be rotated in the opposite direction and then "reconnected" to the program without affecting the current window adjustment.

The FRONT (F) and BACK (B) commands specify window endpoint positions in milliseconds $(M)$, seconds (S), or sound samples relative to one of four base positions: the beginning of the file, the end of the file $(\mathrm{Z})$, the current position of the front of the window $(F)$, or the current position of the back of the window (B). For example: F 5M, front of window to $5 \mathrm{msec}$ from start of file; B 1.5SF, back of window $1.5 \mathrm{sec}$ after the front of the window; F $-80 \mathrm{~F}$, front of window 80 samples before the current window starting position; B Z, back of window to end of file. These commands are often used to make gross adjustments and for resetting a specific window position.

While in audio mode, typing control-F positions the front of the window to the sample being played at that instant. Control-B positions the back of the window in a similar manner. These commands are especially convenient for picking out a word in a long sound segment and for eliminating silence from the beginning and end of a file. Fine adjustments might then be performed with the reference knobs. An experienced SPLIT operator will utilize all three editing control mechanisms interchangeably in order to quickly home in on the exact segment of interest. 


\section{Using Multiple Windows}

SPLIT can simultaneously keep track of nine playback windows which may be either from the same or from different sound files. Only one window is actively edited at any instant. The user assigns an identification number from 1 to 9 to each window/file specification. Switching windows is simple: Just type the window number on the terminal. Rapid comparisons of different sound segments can be easily performed in this way.

The MOVE TO (M) command is a convenient method for associating two or more windows with one file. This is often useful because it enables a sound segment to be inspected alone and in a larger context without the need for continual window adjustments. For example, M 53 causes window/file specification 3 to be assigned the number " 5 ." Now either window may be selected and modified without affecting the position and size of the other one.

Window number 0 is reserved for output files, that is, files that are created during an editing session. The WRITE (W) command appends the active playback window to the output file. In recording mode, the INPUT command records sound into the output file. The MOVE TO command may be used to move the output file to a playback window (example: M 1 0 ) and to create a new output file (example: $M 01$ ).

\section{Software Organization}

The main program for SPLIT requires $8 \mathrm{k}$ of core (including temporary buffer space) and uses several independent modules (see Figure 5):

(1) $O S / 8$ is a DEC operating system for the PDP-8. It runs on computers with $8 \mathrm{k}$ or more of memory and requires 256 words of core on a permanent basis.

(2) RCS (Research Control System) is a programming system which provides higher level $\mathrm{I} / 0$ facilities than $\mathrm{OS} / 8$ for assembly language programs. It occupies one memory field (4k). RCS also has a floating-point interpretive package which uses an additional $1.5 \mathrm{k}$ of core (a modification of DECUS Program 8-594). The floatingpoint routines need not be permanently core resident.

(3) DTALK D/A converts data in OS/8-compatible files from an RK8-E disk system in real time. It occupies

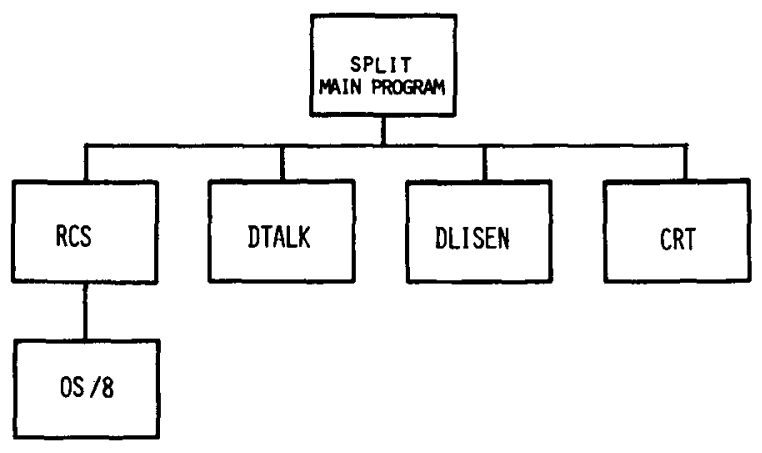

Figure 5. SPLIT software.
768 words of core and uses an additional $4 \mathrm{k}$ memory field for buffer space.

(4) DLISEN A/D converts data and stores it on an RK8-E disk system in real time in OS/8 compatible file format. The subroutine requires 512 words of storage plus a $4 \mathrm{k}$ field for buffer space.

(5) CRT is a set of point plot refresh display functions for an oscilloscope display controlled by the AX08 lab peripheral. Pictures are represented by lists of $(\mathrm{X}, \mathrm{Y})$ point coordinates. The package uses 1,280 words of core (much of this is not needed for SPLIT). Included are subroutines to insert lines and characters into lists.

DTALK and DLISEN can be rewritten to enable other mass storage devices and $A / D-D / A$ peripherals to be used with SPLIT. CRT can be modified to utilize other graphics displays. The maximum recording rate $(21 \mathrm{kHz})$ and playback rate $(24 \mathrm{kHz})$ are software limited on our system. Faster transfer rates can be attained with the use of more complex routines in DTALK and DLISEN. The maximum file length $(800,000$ samples) is also a software limitation, set by the length of a "logical" disk on the system (as opposed to the 1.6-million-word physical disk length). The AX08 lab peripheral actually processes 9-bit samples, but we are currently ignoring the low order bit in order to have samples which fit into the 8-bit byte size of an IBM 360 computer. The 9-bit accuracy can be easily implemented. (Files may be transferred between the PDP-8/E and a $360 / 67$ via magnetic tape or a phone line.)

\section{DISCUSSION}

A large problem in designing real-time interactive systems is the specification of the man-machine interface. We desire flexible programs with many options but want systems to be easy to use and learn. After reading a SPLIT reference manual (available from the second author) and a few hours of on-line instruction, people with little or no computer experience have been able to use SPLIT productively. With practice, an experimenter can become adept in processing a large number of stimuli in a short time. SPLIT is part of an integrated laboratory software system which includes a real-time OS/8 BASIC experimental control system with sound presentation and recording facilities.

\section{REFERENCES}

Anronson, D., Grupsmith, E., \& Ohman, D. S.I.M.P.L.E. control and measurement of auditory events. Behavior Research Methods \& Instrumentation, 1975, 7, 125-130.

KNIGET, J. L., JR., \& KartowTTz, B. H. A minicomputer method for generating dichotic word pairs. Behavior Research Methods \& Instrumentation, 1973, 5, 231-234.

Nakatani, L. H. Computer-aided signal handling for speech research. Journal of the Acoustical Society of America, 1977, 61, 1056-1062.

Poltrock, S. E., \& Mathews, N. N. A system for computer control of auditory stimuli. Behavior Research Methods \& Instrumentation, 1976, 8, 197-199. 